\title{
TRILATERAL FILTERING FOR BIOMEDICAL IMAGES
}

\author{
Wilbur C.K. Wong ${ }^{\dagger}$, Albert C.S. Chung ${ }^{\dagger}$, Simon C.H. Yu ${ }^{\ddagger}$ \\ ${ }^{\dagger}$ Department of Computer Science, The Hong Kong University of Science and Technology, HK. \\ ${ }^{\ddagger}$ Department of Diagnostic Radiology and Organ Imaging, The Prince of Wales Hospital, HK.
}

\begin{abstract}
Filtering is a core operation in low level computer vision. It is a preliminary process in many biomedical image processing applications. Bilateral filtering has been applied to smooth biomedical images while preserving the edges. However, to avoid oversmoothing structures of sizes comparable to the image resolutions, a narrow spatial window has to be used. This leads to the necessity of performing more iterations in the filtering process. In this paper, we propose a novel filtering technique namely trilateral filter, which can achieve edge-preserving smoothing with a narrow spatial window in only a few iterations. The experimental results have shown that our novel method provides greater noise reduction than bilateral filtering and smooths biomedical images without over-smoothing ridges and shifting the edge locations, as compared to other noise reduction methods.
\end{abstract}

\section{INTRODUCTION}

Filtering is a preliminary process in many biomedical image processing applications. It is a fundamental operation in low level computer vision, which is aimed at restoring a noise-corrupted image to its noiseless counterpart. Any post-processing tasks, such as visualization and segmentation, may benefit from the reduction of noise. Diffusion equations with scalar-valued and tensor-valued diffusivities have been applied to magnetic resonance (MR) imaging and 3D rotational angiography (RA) [1], [2], [3] for edgepreserving smoothing. MR angiography (MRA) denoising with adaptive filtering in the Fourier domain has been proposed in [4]. Non-linear noise reduction techniques in computed tomography (CT) imaging have been investigated in [5].

In this paper, we present a novel filtering method for biomedical images, namely trilateral filtering. The method works along the same lines as bilateral filtering [6], integrating geometric, photometric and local structural similarities, to achieve edgepreserving smoothing. It is simple to implement and is applicable to multi-dimensional signals. It uses a narrow spatial window (3 pixels in each dimension) and takes only a few iterations (3 iterations in all the experiments conducted in this work) in the smoothing process.

This paper is organized as follows: Section 2 briefly introduces the bilateral filter, as the fundamental of our trilateral filtering. The formulation of the trilateral filter follows. In Section 3, sensitivity analyses of the new method as well as experimental results on 3D numerical phantoms, 2D and 3D clinical datasets and a 3D MR imaging phantom are presented. Conclusions are drawn in Section 4.

\section{FUNDAMENTAL AND METHOD}

In this section, we present the fundamental and the method formulation of the new filtering technique. Section 2.1 introduces bilateral filtering, as the preliminary of our trilateral filtering. The formulation of the trilateral filter is given in Section 2.2. In Section 2.3 , the methodology to extract the local structural information is discussed.

\subsection{Bilateral Filtering}

Bilateral filtering, representing a large class of non-linear filters proposed by Tomasi et al. [6], is a non-iterative and local approach to edge-preserving smoothing. A filtered image is obtained by replacing the intensity value of each pixel with an average value weighted by the geometric and photometric similarities between neighboring pixels within a spatial window. The bilateral filtering can be summarized in the following discrete formulation:

$$
\vec{I}^{*}(\vec{x})=\frac{1}{k(\vec{x})} \sum_{\vec{\xi} \in \mathcal{N}_{\vec{x}}} \vec{I}(\vec{\xi}) \cdot c(\vec{\xi}, \vec{x}) \cdot s(\vec{I}(\vec{\xi}), \vec{I}(\vec{x})),
$$

where $\vec{x}$ and $\vec{\xi}$ are spatial coordinates, $\vec{I}$ is the noisy image, $\vec{I}^{*}$ is the filtered image, $\mathcal{N}_{\vec{x}}$ defines the spatial window around the pixel at $\vec{x}, k(\vec{x})$ is the normalization constant which assures the weights $c(\cdot) \cdot s(\cdot)$ are added up to 1 within $\mathcal{N}_{\vec{x}}$, the functions $c$ and $s$ measure the geometric and photometric similarities between neighborhoods respectively. For the definitions of the two functions, see [6].

The concept of bilateral filtering has been investigated in a recent publication. Barash [7] revealed that bilateral filtering is a non-iterative method only if a wide spatial window is used (15 pixels in each dimension). However, a wide spatial window may over-smooth sharp ridges and gutters in the image as indicated in [8]. Therefore, it is necessary to strike a balance between the size of the spatial window and the number of iterations needed to be performed in bilateral filtering.

\subsection{Trilateral Filtering for Biomedical Images}

Sharp ridges and gutters are commonly found in biomedical images, such as nested vessels in digital subtraction angiography (DSA) and 3D angiography, and folded gray and white matters in brain MR imaging. Therefore, a narrow spatial window, say, 3 pixels in each dimension, should be used in order to avoid oversmoothing structures of sizes comparable to the image resolutions. This leads to the necessity of performing more iterations in the filtering process. 
In this paper, we propose a novel filtering method for biomedical images, which works along the same lines as the bilateral filter, it takes not only the geometric and photometric similarities into account, but also, the local structural similarity to smooth the images with a narrow spatial window while preserving the edges. Local structural information is used to determine inhomogeneity in the images. On one hand, low-pass filtering is performed in the homogeneous regions. On the other hand, smoothing along edges is achieved by considering the geometric, photometric and local structural orientation similarities between neighboring pixels in the inhomogeneous regions. We found that this new approach provides greater noise reduction than bilateral filtering with a 3pixel-width spatial window (cf. Section 3).

Because of the use of the three similarities in the filtering process, we name this novel method trilateral filtering. The trilateral filtering is expressed as follows:

$$
\vec{I}^{(t+1)}(\vec{x})=\frac{1}{k(\vec{x})} \sum_{\vec{\xi} \in \mathcal{N}_{\vec{x}}} \vec{I}^{(t)}(\vec{\xi}) \cdot w(\vec{\xi}, \vec{x}, t),
$$

where

$$
\begin{aligned}
& w(\vec{\xi}, \vec{x}, t)=(1-a(\vec{x})) \cdot c(\vec{\xi}, \vec{x})+ \\
& a(\vec{x}) \cdot c(\vec{\xi}, \vec{x}) \cdot s\left(\vec{I}^{(t)}(\vec{\xi}), \vec{I}^{(t)}(\vec{x})\right) \cdot \sum_{i=1}^{D-1} d_{i}(\vec{\xi}, \vec{x}),
\end{aligned}
$$

$t$ is a time variable, $a(\vec{x}) \in[0,1]$ is the regularized local signal amplitude (cf. Section 2.3) of the pixel at $\vec{x}, a \rightarrow 0$ in the homogeneous regions, the functions $c$ and $s$ are defined as in the bilateral filter, $D$ is the dimensionality of the image $\vec{I}$ and the function $d_{i}$ measures the similarity of the rank $i$ local structural orientation between the pixels at $\vec{\xi}$ and $\vec{x}$. Rank 1 orientation refers to the principal direction of a linear structure, whereas rank 2 orientation refers to one of the principal directions of a planar structure (cf. Section 2.3). The function $d_{i}$ is defined as follows:

$$
d_{i}(\vec{\xi}, \vec{x})=\exp \left(-\frac{\delta^{2}\left(\vec{\xi}-\vec{x}, \hat{e}_{i}\right)}{2 \sigma^{2}}\right),
$$

where

$$
\delta(\vec{u}, \vec{v})=1-\left|\frac{\vec{u} \cdot \vec{v}}{\|\vec{u}\|\|\vec{v}\|}\right|,
$$

$\hat{e}_{i}$ is the direction of rank $i$ orientation and the parameter $\sigma$ is chosen based on the desired amount of orientation discrepancy filtering between neighborhoods. $\delta \rightarrow 0$ if the angle between $\vec{u}$ and $\vec{v}$ is $0^{\circ} / 180^{\circ}$, therefore, $d_{i} \rightarrow 1$ if the pixel at $\vec{\xi}$ locates along the direction $\hat{e}_{i}$ with respect to the pixel at $\vec{x}$.

In other words, the trilateral filter produces a smoothed image by weighting the intensity value of each pixel within the narrow spatial window. Weights are either defined by the geometric similarity, $c(\cdot)$, in the homogeneous regions or defined trilaterally by the geometric, photometric and local structural orientation similarities, $c(\cdot) \cdot s(\cdot) \cdot \sum_{i} d_{i}(\cdot)$, in the inhomogeneous regions.

\subsection{Extraction of Local Structural Information}

The local structural information is obtained from the eigen decomposition of orientation tensors as described in a previous work [9],

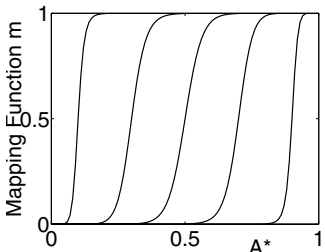

(a)

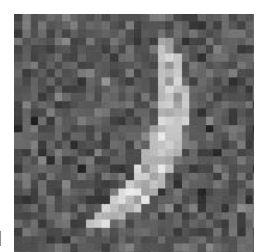

(b)

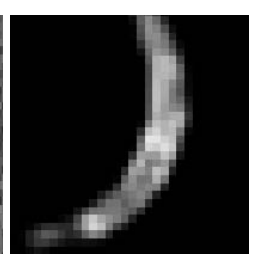

(c)
Fig. 1. Local signal amplitude regularization. (a) Mapping function $m$ as a function of $A_{\vec{x}}^{*} \in[0,1], q=4$, with five different values of p: 0.1 (leftmost curve), 0.3, 0.5, 0.7 and $0.9 ;(b)$ a portion of a slice image from a numerical phantom; and (c) regularized local signal amplitudes $a, p=0.3$ and $q=4$ in the regularization process. It is evident that $a \rightarrow 0$ (dark) in the background region, while $a \rightarrow 1$ (bright) if the pixel at $\vec{x}$ is located near the edges of the bright structure. Therefore, it can be used to determine the inhomogeneity in an image

in which it is demonstrated that the use of the orientation tensor gives more reliable local structural information than the use of the Hessian matrix. The vectors $\hat{e}_{1}$ and $\hat{e}_{2}$ are the eigenvectors corresponding to the first and second smallest eigenvalues in the decomposition. The Frobenius norm of the orientation tensor (i.e., $\sqrt{\sum_{i=1}^{D} \lambda_{i}{ }^{2}}$, where $\lambda_{i}, i=1,2, \ldots, D$, are the eigenvalues of the tensor in a $D$-dimensional space) defines the local signal amplitude $A_{\vec{x}} \in[0,+\infty)$. Regularization is achieved by a mapping function $m$, which maps the normalized local signal amplitude $A_{\vec{x}}^{*} \in[0,1]$ to $[0,1]$, as follows:

$$
\begin{aligned}
a(\vec{x})=m\left(A_{\vec{x}}^{*}, p, q\right) & = \\
& \frac{\left(A_{\vec{x}}^{*} \cdot(1-p)\right)^{q}}{\left(A_{\vec{x}}^{*} \cdot(1-p)\right)^{q}+\left(\left(1-A_{\vec{x}}^{*}\right) \cdot p\right)^{q}},
\end{aligned}
$$

where $p \in[0,1]$ and $q$ are positive constants. Figure 1(a) shows the mapping function $m$ as a function of $A_{\vec{x}}^{*}, q=4$, with five different values of $p$. Figure 1(b) shows a portion of a slice image from a numerical phantom (see Section 3 for details). Figure 1(c) shows the regularized local signal amplitudes $a, p=0.3$ and $q=$ 4 in the regularization process. It is evident that $a \rightarrow 0$ (dark) in the background region, while $a \rightarrow 1$ (bright) if the pixel at $\vec{x}$ is located near the edges of the bright structure. Therefore, the regularized local signal amplitudes $a$ can be used to determine the inhomogeneity in an image.

\section{RESULTS AND DISCUSSION}

A $64 \times 64 \times 64$ voxels numerical phantom has been built to evaluate the performance of the trilateral filter. The phantom is shown in Figure 2(a), which is a pipe ( 5 voxels in diameter) in a 3D space with its centerline aligned with a cubic B-spline curve. In order to study the capability to smooth an image with a narrow spatial window $(3 \times 3 \times 3$ voxels), we have applied the trilateral filter $(\mathrm{TF})$ and the bilateral filter (BF) to the numerical phantom at signal level equals 5 . The signal level is defined as follows:

$$
\text { Signal Level }=\frac{Q}{\sigma_{N}}
$$

where $Q=255$ is the intensity value of the noiseless pipe and $\sigma_{N}$ is the standard deviation of an additive white Gaussian noise. The 


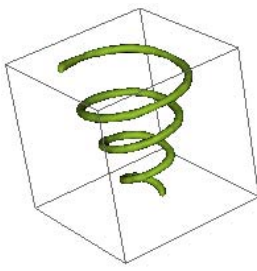

(a) Surface model

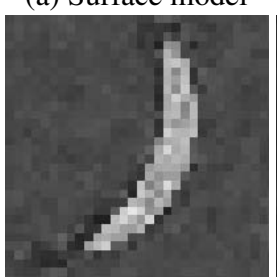

(d) $\mathrm{AF}$

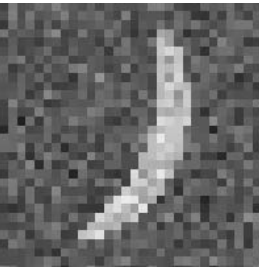

(b) Original

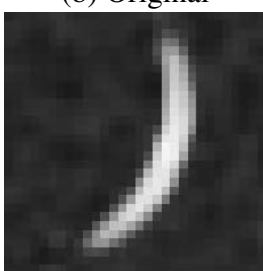

(e) EED

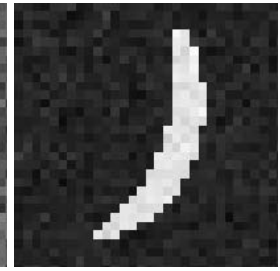

(c) $20 \mathrm{NEX}$

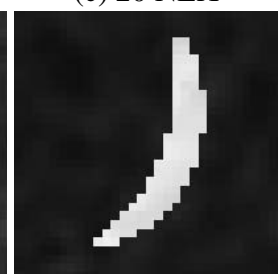

(f) TF
Fig. 2. Numerical phantom. (a) 3D surface model; (b) a portion of a slice image from the phantom; smoothed images with (c) 20 $N E X,(d) A F(e) E E D$ and $(f) T F$

signal level 5 implies $\sigma_{N}=255 / 5=51$. We found that there is $93.96 \%$ decrease in the mean square error (MSE) for TF, in contrast to $59.20 \%$ decrease in MSE for BF, using a single iteration. These results have shown that TF provides better image denoising than BF, using a narrow (3-pixel-width) spatial window.

Furthermore, we have conducted four noise sensitivity analyses, in which the increases in (a) signal-to-noise ratio (SNR), (b) contrast-to-noise ratio (CNR), (c) MSE and (d) deviation in gradient direction after the application of different denoising methods are studied. SNR and CNR are defined in the following:

$$
\begin{gathered}
\mathrm{SNR}=\frac{\overline{Q_{P}}}{\sigma_{B}}, \\
\mathrm{CNR}=\left(\frac{\overline{Q_{P}}-\overline{Q_{B}}}{\sigma_{B}}\right)^{2},
\end{gathered}
$$

where $\overline{Q_{P}}$ and $\overline{Q_{B}}$ are the mean intensity values of the pipe and the background respectively, and $\sigma_{B}$ is the sample standard deviation of the noise in the background. Deviation in gradient direction is calculated according to Equation 5, taking the truth gradient vector and the computed gradient vector from the denoised image as arguments. We have compared TF with three other denoising methods, viz. 20 NEX (averaging), adaptive filtering (AF) [4] and edge-enhancing anisotropic diffusion (EED) [3], where AF and EED are edge-preserving noise reduction techniques.

Figure 2(b) shows a portion of a slice image from the phantom at signal level equals 5 . Figures 2(c)-(f) show the filtered images obtained with 20 NEX, AF, EED and TF respectively. Figure 3 shows the results of the noise sensitivity analyses. Increases are measured in $\mathrm{dB}$ according to this formula:

$$
\text { Increase in } \mathrm{dB}=20 \cdot \log _{10} \frac{\text { New Value }}{\text { Old Value }} \text {. }
$$

It is noted that TF gives the largest amount of reduction in MSE and the greatest increase in both SNR and CNR amongst the other methods. Further, TF provides better gradient direction restoration than 20 NEX if the signal level $\geq 5$.

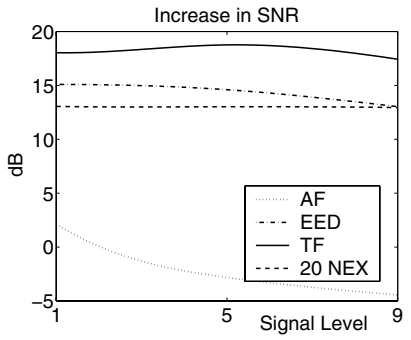

(a)

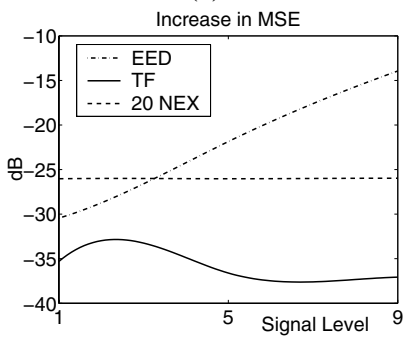

(c)

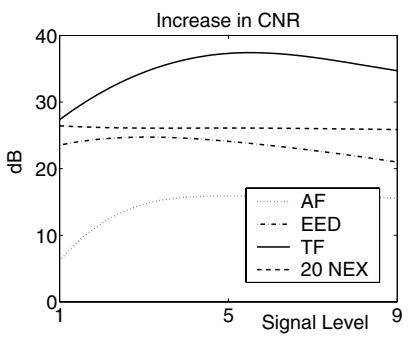

(b)

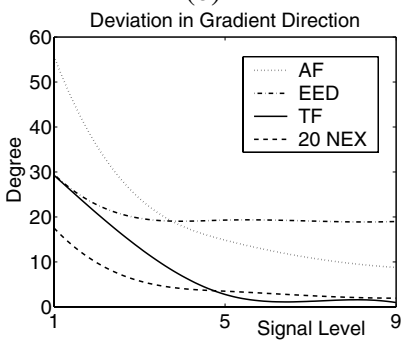

(d)
Fig. 3. Results of noise sensitivity analyses. Increases in (a) SNR, (b) CNR and (c) MSE; and (d) deviation in gradient direction at different signal levels

In addition to the numerical phantom, we have applied TF to 2D and 3D biomedical datasets and a 3D MR imaging phantom. Figure 4 shows filtered images of a 2D DSA obtained with BF and TF. Figure 5 shows denoised slice images of a 3D RA produced by AF, EED and TF. Figure 6 shows smoothed slice images of a 3D T1 MR imaging phantom from BrainWeb ${ }^{1}$ obtained with AF, EED, BF and TF. It is evident that: TF gives a greater noise reduction than $\mathrm{BF}$ in the vascular regions, see Figures 4(d)-(f), and smooths the DSA image without over-smoothing ridges, as found in the BF denoised image, pointed by the arrows in Figures 4(b) and 4(e); TF produces a smoothed 3D RA image without shifting the edge locations as compared to EED, indicated by the arrow in Figure 5(e); and TF gives smoother solution in homogeneous regions, while produces sharper edges than the other methods as illustrated in the T1 MR imaging phantom (see Figure 6).

\section{CONCLUSION}

We have presented a novel denoising method for biomedical images, namely the trilateral filter. The method integrates the geometric, photometric and local structural similarities to filter the images. It replaces the intensity value at each pixel with an average value weighted by the three similarities between neighboring pixels within a narrow spatial window.

In order to evaluate the performance of the trilateral filter, we have conducted several experiments on 3D numerical phantoms, 2D and 3D biomedical datasets and a 3D MR imaging phantom. The experimental results have shown that our novel method produces greater noise reduction than the bilateral filtering and smooths the images without over-smoothing ridges and shifting the edge locations, as compared to other edge-preserving noise reduction methods.

${ }^{1}$ A simulated brain database from Montréal Neurological Institute, McGill University (www.bic.mni.mcgill.ca/brainweb/) . 


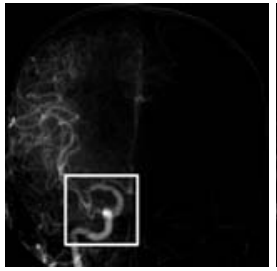

(a) Original

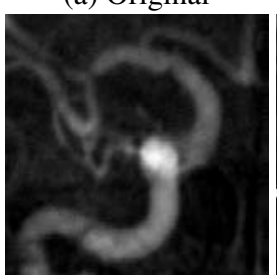

(d) Original

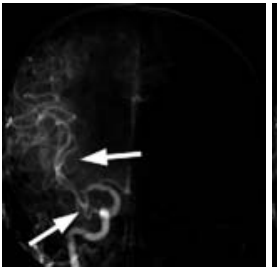

(b) $\mathrm{BF}$

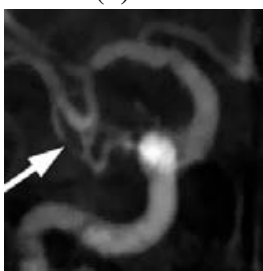

(e) $\mathrm{BF}$

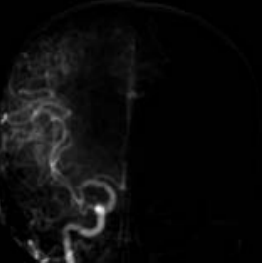

(c) $\mathrm{TF}$

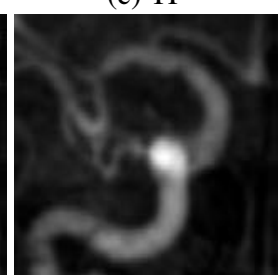

(f) $\mathrm{TF}$
Fig. 4. DSA. (a) Original image; denoised images with (b) BF and (c) TF; and (d)-(f) closeup of the images $(a)-(c)$

In this paper, we have demonstrated an application of the trilateral filter. We expected that this new method will not be restricted to biomedical images. It could be applicable to other image types, e.g., natural scenes, artwork or spatial-temporal images.

\section{REFERENCES}

[1] G. Gerig, O. Kübler, R. Kikinis, and F. A. Jolesz, "Nonlinear anisotropic filtering of MRI data," TMI, vol. 11, no. 2, pp. 221-232, 1992.

[2] K. Krissian, "Flux-based anisotropic diffusion applied to enhancement of 3-D angiogram," TMI, vol. 21, no. 11, pp. 14401442,2002

[3] E. Meijering, W. Niessen, J. Weickert, and M. Viergever, "Diffusion-enhanced visualization and quantification of vascular anomalies in three-dimensional rotational angiography: Results of an in-vitro evaluation," MedIA, vol. 6, no. 3, pp. 215-233, 2002

[4] C. F. Westin, L. Wigström, T. Loock, L. Sjöqvist, R. Kikinis, and $\mathrm{H}$. Knutsson, "Three-dimensional adaptive filtering in magnetic resonance angiography," JMRI, vol. 14, pp. 6371, 2001.

[5] G. Fernández, H. Bischof, and R. Beichel, "Nonlinear filters on 3D CT imaging - bilateral filter and mean shift filter," in Computer Vision Winter Workshop, 2003.

[6] C. Tomasi and R. Manduchi, "Bilateral filtering for gray and color images," in ICCV, 1998, pp. 839-846.

[7] D. Barash, "A fundamental relationship between bilateral filtering, adaptive smoothing, and the nonlinear diffusion equation," PAMI, vol. 24, no. 6, pp. 844-847, 2002.

[8] P. Choudhury and J. Tumblin, "The trilateral filter for high contrast images and meshes," in Eurographics Symposium on Rendering, 2003.

[9] W. C. K. Wong, A. C. S. Chung, and S. C. H. Yu, "Local orientation smoothness prior for vascular segmentation of angiography," 2003, Submitted to ECCV 2004.

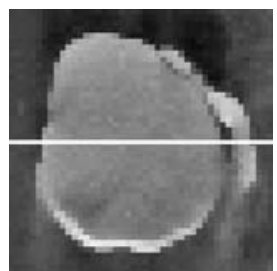

(a) Original

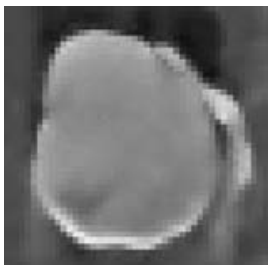

(b) $\mathrm{AF}$

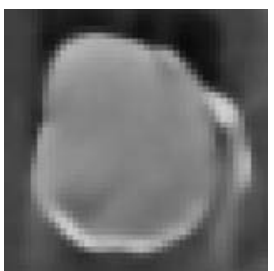

(c) EED

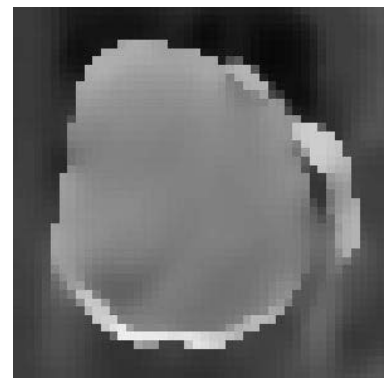

(d) $\mathrm{TF}$

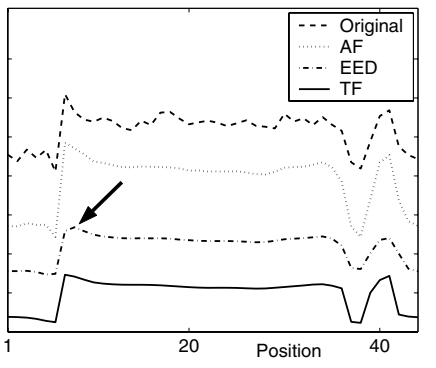

(e) Profile
Fig. 5. $3 D R A$. (a) A slice image from the dataset; filtered images with (b) $A F$, (c) EED and (d) TF; and (e) intensity profile of the images $(a)-(d)$ at the scanline defined in $(a)$

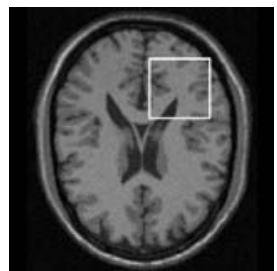

(a) Original

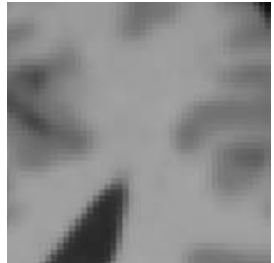

(d) EED

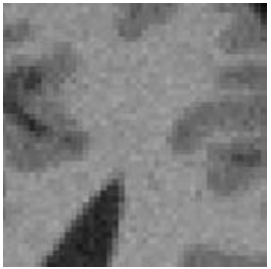

(b) Closeup

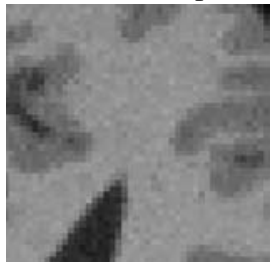

(e) $\mathrm{BF}$

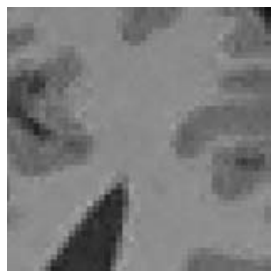

(c) $\mathrm{AF}$

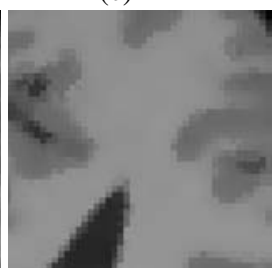

(f) $\mathrm{TF}$
Fig. 6. $T 1 M R$ imaging. (a) An original slice image; (b) closeup of the slice image; smoothed images with (c) $A F,(d) E E D,(e) B F$ and $(f) T F$ 